\title{
Gravitational instability in suspension flow
}

\author{
By ILEANA C. CARPEN AND JOHN F. BRADY \\ Division of Chemistry and Chemical Engineering, California Institute of Technology, \\ Pasadena, CA 91125, USA
}

(Received 17 June 2002 and in revised form 26 August 2002)

The gravity-driven flow of non-neutrally buoyant suspensions is shown to be unstable to spanwise perturbations when the shearing motion generates a density profile that increases with height. The instability is simply due to having heavier material over light - a Rayleigh-Taylor-like instability. The wavelength of the fastest growing disturbance is on the order of the thickness of the suspension layer. The parameters important to the problem are the angle of inclination of the layer relative to gravity, the relative density difference between the particles and the fluid, the ratio of the particle size to the thickness of the layer and the bulk volume fraction of particles. The instability is illustrated for a range of these parameters and shown to be most pronounced at intermediate values thereof. This instability mechanism may play an important role in pattern formation in multiphase flows.

\section{Introduction}

Particulates are an integral part of many industrial processes, and whether in the form of suspensions or dry granular flows, they present an engineering challenge that has so far been met empirically and with only partial success. Thus, there has been interest in the last few decades in analysing the complex behaviours of granular and granular-fluid flow, but the field remains rich with unexplained problems, with new phenomena being continually discovered.

Granular media can exhibit complex behaviours, being solid, liquid, or gas-like depending on the situation, and sometimes exhibiting multiple states (e.g. see the reviews by Jaeger, Nagel \& Behringer 1996a, b and Savage 1984). Among the interesting facets of granular flow behaviour is a large set of instabilities, including oscillons formed in vertically vibrated containers, e.g. see Umbanhowar, Melo \& Swinney (1996), segregation of granular media in mixers driven by such differences in particle properties as size, density and shape (Hill \& Kakalios 1995), fingering instabilities in suspensions and dry granular flows (Lange et al. 1998; Pouliquen, Delour \& Savage 1997), segregation of neutrally buoyant particles in suspensions (Tirumkudulu, Mileo \& Acrivos 2000), wave patterns in sand (Fried, Shen \& Thoroddsen 1998), and longitudinal vortices in granular flows (Forterre \& Pouliquen 2001). Analysing these instabilities and patterns can lead to crucial insights into granular and granular-fluid flow behaviour.

In many situations, the particles are heavier than the suspending fluid, whether liquid or gas, and the resulting density variations can lead to flow, as in the experiments of Forterre \& Pouliquen (2001) for granular flow down an inclined plane, or the fingering instability in a sedimenting suspension analysed by Völtz, Pesch \& Rehberg (2001). In flows with heavy particles, rapid shearing motion adjacent to a boundary can create a region of low density that supports the weight of a denser, slower 


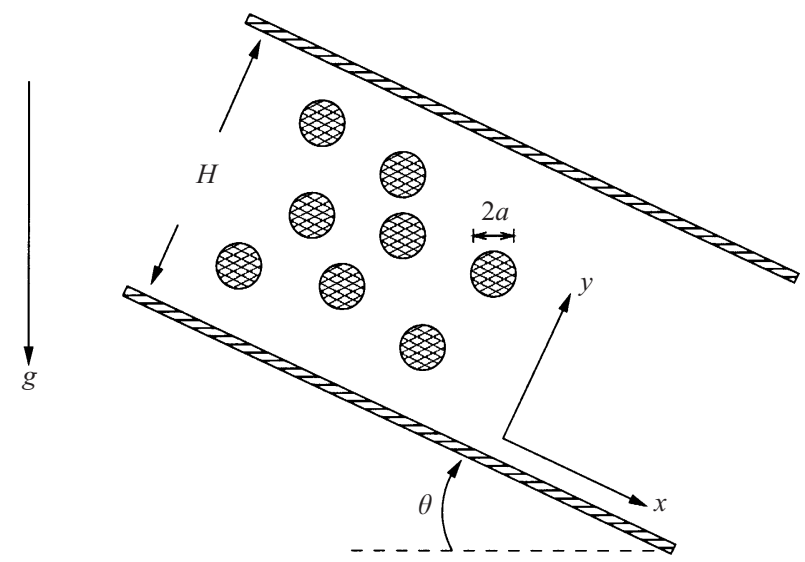

FIGURE 1. Gravity-driven flow of a suspension through an inclined channel of height $H$. The inclination angle $\theta$ ranges between $0^{\circ}$ and $90^{\circ}$, from pure sedimentation to vertical flow. The direction parallel to the channel walls (the flow direction) is taken to be $x$.

shearing layer (cf. figure 5). This adverse density stratification can then be unstable to spanwise (or longitudinal) disturbances, similar to the classic Rayleigh-Taylor instability: a local increase in density (or particle fraction) will fall due to gravity and drag down material from above which is even denser, further enhancing the perturbation. This instability mechanism appears to be new and generic, although the precise growth rates and wavelengths will depend on the nature of the suspension or granular flow. This instability may be operative in several granular and suspension flows and may be responsible for a variety of patterns observed in these systems.

To illustrate this basic instability mechanism we consider the situation of a heavyparticle viscous suspension flowing through an inclined channel and examine its stability to perturbations spanwise to the flow. In the gravity-driven flow, the particle volume fraction, and therefore the density, can increase with height in the velocity gradient direction due to a balance between shear-induced particle migration and buoyancy and thus be unstable. Section 2 defines the problem and introduces the governing equations. The base state showing the adverse density stratification is discussed in $\S 3$, and its linear stability to spanwise perturbations is considered in $\S 4$, where the motion is seen to be unstable at all wavelengths, with a maximum growth rate at a wavelength on the order of the suspension layer thickness. Concluding remarks are given in $\S 5$.

\section{The governing equations}

The problem considered is gravity-driven flow of a suspension down an inclined channel as illustrated in figure 1 . The system is modelled as a continuum viscous suspension and we use the suspension-balance model of Nott \& Brady (1994) as modified by Morris \& Brady (1998). In the following we ignore inertial effects, an acceptable assumption for low particle and geometry Reynolds numbers, and the suspension as a whole is incompressible. Thus, conservation of mass and momentum for the suspension are

$$
\begin{gathered}
\nabla \cdot\langle\boldsymbol{u}\rangle=0, \\
\nabla \cdot\langle\boldsymbol{\sigma}\rangle+\rho \boldsymbol{g}=0,
\end{gathered}
$$


where $\rho=\rho_{f}+\Delta \rho \phi$ and $\Delta \rho=\rho_{p}-\rho_{f}$. The subscripts $p$ and $f$ denote the particle and fluid phases, respectively. We also have mass and momentum conservation for the particle phase:

$$
\begin{gathered}
\frac{\partial \phi}{\partial t}+\nabla \cdot\langle\boldsymbol{u}\rangle_{p} \phi=0, \\
\nabla \cdot\langle\boldsymbol{\sigma}\rangle_{p}+\Delta \rho \phi \boldsymbol{g}-\frac{9}{2} \frac{\eta}{a^{2}} \frac{\phi}{f(\phi)}\left(\langle\boldsymbol{u}\rangle_{p}-\langle\boldsymbol{u}\rangle\right)=0,
\end{gathered}
$$

where $f(\phi)$ is the hindered settling function, and $\phi$ denotes the volume fraction of particles of size $a$. The stress $\langle\boldsymbol{\sigma}\rangle$ and the velocity $\langle\boldsymbol{u}\rangle$ are suspension-averaged quantities (particle-phase averages if accompanied by the subscript $p$ ). In addition to the mass and momentum conservation equations for the suspension and the particle phases, it is necessary to include an energy balance, bringing in the suspension temperature. In an inhomogeneous flow, the stress can be finite even in regions of vanishing shear rate due to a possibly non-zero local fluctuational motion $\boldsymbol{u}_{p}^{\prime}=\boldsymbol{u}_{p}-\langle\boldsymbol{u}\rangle_{p}$. The suspension temperature $T=\left\langle\boldsymbol{u}_{p}^{\prime} \cdot \boldsymbol{u}_{p}^{\prime}\right\rangle$ introduces the necessary non-locality for the stress. For these inertia-free flows the energy balance takes the form

$$
\langle\boldsymbol{\sigma}\rangle_{p}:\langle\mathbf{e}\rangle-\eta \frac{\alpha(\phi)}{a^{2}} T+\eta \nabla \cdot \kappa(\phi) \nabla T=0,
$$

where $\langle\mathbf{e}\rangle$ is the average rate-of-strain tensor, and the flux of fluctuational energy has been modelled by a Fourier-type law. For convenience in notation, $\langle\cdot\rangle$, denoting an average, will be dropped from all further equations.

The constitutive relations for the particle and suspension stresses are taken to be those appropriate for a viscous suspension (Morris \& Brady 1998):

$$
\begin{gathered}
\boldsymbol{\sigma}_{p}=-\Pi \boldsymbol{I}+2 \eta \eta_{p}(\phi) \boldsymbol{e}, \\
\boldsymbol{\sigma}=-p_{f} \boldsymbol{I}+2 \eta \boldsymbol{e}+\boldsymbol{\sigma}_{p}=-\left(p_{f}+\Pi\right) \boldsymbol{I}+2 \eta \eta_{s}(\phi) \boldsymbol{e},
\end{gathered}
$$

where $\Pi$ is the particle-phase pressure

$$
\Pi=\eta a^{-1} p(\phi) \sqrt{T},
$$

$p_{f}$ is the average pressure in the fluid, and $\eta_{p}$ and $\eta_{s}$ are the relative viscosities for the particle phase and the suspension, respectively.

To close the equation set we also need to specify the volume fraction dependence of the functions $p, \eta_{s}, \alpha, \kappa$ and $f$; as in Morris \& Brady (1998), we take these functions to be

$$
\begin{gathered}
p(\phi)=\eta_{p}(\phi), \\
\eta_{s}(\phi)=1+\eta_{p}(\phi)=\left(1-\phi / \phi_{m}\right)^{-2}, \\
\alpha(\phi)=k_{\alpha} \eta_{p}(\phi) / \phi, \\
\kappa(\phi)=k_{\kappa} \eta_{p}(\phi), \\
f(\phi)=(1-\phi)^{-5},
\end{gathered}
$$

with $k_{\alpha}=0.815$ and $k_{\kappa}=0.8$. The maximum volume fraction is taken to be $\phi_{m}=$ 0.68 . In a homogeneous shear flow with constant shear rate $\dot{\gamma},\langle\sigma\rangle_{p}=\eta_{p} \dot{\gamma}$ and equation (2.3) gives an uniform suspension temperature $T=(\dot{\gamma} a)^{2} \alpha(\phi) / \eta_{p}(\phi)$ as expected. 
For a more complete description of the model chosen and the reasoning behind its derivation, the reader is referred to Nott \& Brady (1994), Morris \& Brady (1998) and references contained therein.

\section{The base state}

The parameters that govern the flow are: the angle of inclination with respect to the horizontal, $\theta$, the ratio of the particle size, $a$, to the channel width, $H$, with $\epsilon=a / H \ll 1$, the bulk volume fraction of particles, $\phi_{b}$, and the density ratio $B=\Delta \rho / \rho_{f}$. The gravitational acceleration $g$ and the fluid viscosity $\eta$ are important in establishing the characteristic velocity, $U_{c}=\rho_{f} g H^{2} / \eta$, but do not appear in the dimensionless groups characterizing the problem and therefore affect only the quantitative behaviour of the system. Gravity affects the qualitative behaviour of the system through the angle of inclination $\theta$.

The base-state flow is unidirectional, parallel to the channel walls, and satisfies the conservation equations (2.1)-(2.3). Using the constitutive relations defined above and taking $(u, v, w)$ as the $(x, y, z)$ components of the suspension velocity, we obtain the following system of dimensionless equations, where the variables are scaled with $y \sim H, u \sim U_{c}$ and $T \sim \epsilon^{2} U_{c}^{2}:$

$$
\begin{gathered}
\frac{\mathrm{d}}{\mathrm{d} y} \eta_{s}(\phi) \frac{\mathrm{d} u}{\mathrm{~d} y}=-(1+B \phi) \sin \theta, \\
\frac{\mathrm{d}}{\mathrm{d} y}(p(\phi) \sqrt{T})=-B \phi \cos \theta, \\
\epsilon^{2} \frac{\mathrm{d}}{\mathrm{d} y} \kappa(\phi) \frac{\mathrm{d} T}{\mathrm{~d} y}-\alpha(\phi) T=-\eta_{p}(\phi)\left(\frac{\mathrm{d} u}{\mathrm{~d} y}\right)^{2} .
\end{gathered}
$$

The velocity, temperature and volume fraction satisfy the boundary conditions

$$
\begin{gathered}
u=0 \quad \text { at } \quad y=0 \quad \text { and } \quad y=1, \\
T=\frac{\eta_{p}(\phi)}{\alpha^{*}(\phi)}\left(\frac{\mathrm{d} u}{\mathrm{~d} y}\right)^{2} \quad \text { at } \quad y=0 \quad \text { and } \quad y=1,
\end{gathered}
$$

and the integral constraint on volume fraction is

$$
\int_{0}^{1} \phi(y) \mathrm{d} y=\phi_{b} .
$$

In (3.2b) $\alpha^{*}(\phi)=20 \alpha(\phi)$ (Morris \& Brady 1998), stating that the fluctuational motion is reduced near a boundary but scales in the same manner as in the bulk. In the base state $u_{p}-u \sim O\left(\epsilon^{2}\right)$, i.e. there is no phase slip.

This system does not lend itself to an analytical solution and was solved numerically. Typical results are shown in figure 2 for a range of channel inclinations and at a density ratio $B=1$, in figure 3 for a range of $B$ at a specific channel inclination and in figure 4 for a range of bulk volume fractions and a range of $\epsilon=a / H$, the ratio of particle size to channel height. In general, the suspension velocity profile is blunted in comparison to the corresponding parabolic Newtonian velocity profile - the variation in the particle volume fraction affects the suspension viscosity, very strongly so in regions of high density such as near the centre of the channel (where $\phi \rightarrow \phi_{m}$ ), and since the shear stress varies almost linearly with position, the shear rate is diminished in those regions. Note that the blunting of the velocity profile 

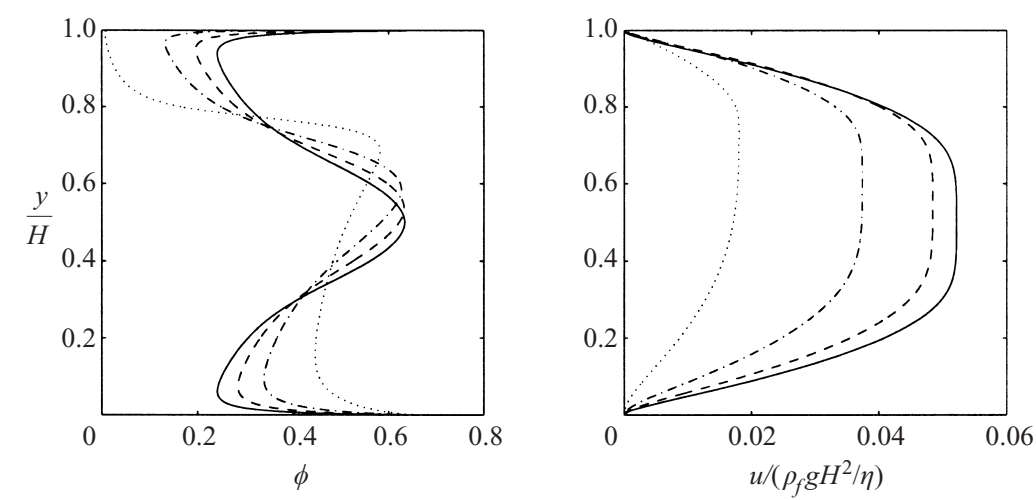

FiguRE 2. Base state particle volume fraction and velocity profiles for $\phi_{b}=0.40, H / a=30.54$ and $B=1: \theta=\left\{30^{\circ}(\cdots), 50^{\circ}(-\cdot-\cdot-), 70^{\circ}(--), 90^{\circ}(\square)\right\}$. Increasing $\theta$ results in increasing symmetry around the centre of the channel.
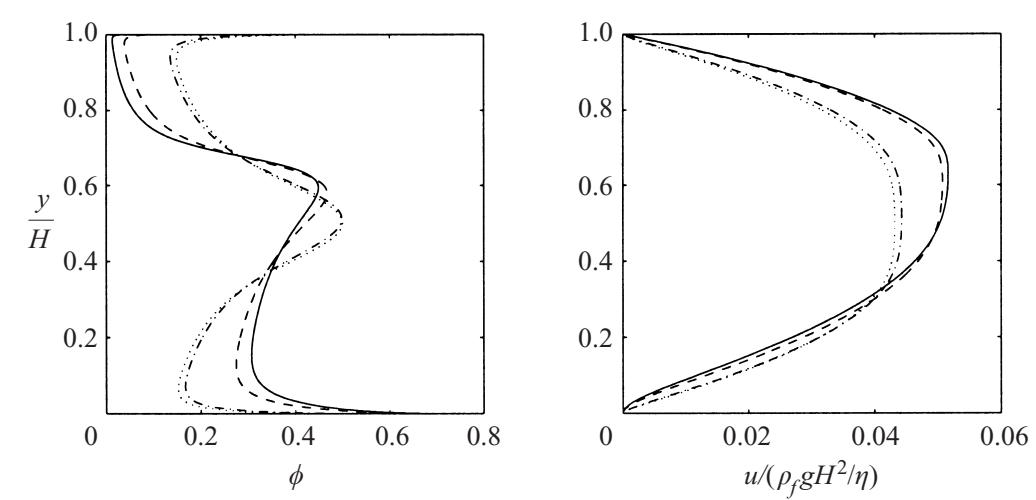

FiguRE 3. Base state volume fraction and velocity profiles for $\phi_{b}=0.27, \theta=45^{\circ}, H / a=18.32$ and $B=\{0.01(\cdots), 0.1(-\cdot-\cdot-), 1(--), 1.4(-)\}$. For small $B$ the velocity grows with increasing $B$. It achieves a maximum for $B \approx 1$, and experiences a slight decrease upon further increase in $B$. Increasing $B$ always results in increasing asymmetry in the velocity and density profiles.

'follows' the maximum in the volume fraction (see figure 3). Decreasing inclination angle, $\theta$, and increasing density ratio, $B$, lead to increasing sedimentation, and in the limits $\theta \rightarrow 0$ or $B \rightarrow \infty$ the particles pack down on the bottom of the channel and flow as a plug (if they flow at all - in the $\theta \rightarrow 0$ limit, the driving force for flow in the $x$-direction is lost and the system becomes a sedimenting one). However, for $\theta \neq 0$ and more physically reasonable density ratios, $B \sim O(1)$, the particle volume fraction varies in a more interesting manner across the channel, as seen in figure 2, reminiscent of the results of Nott \& Brady (1994) and Morris \& Brady (1998) for pressure-driven flow. Similar to the results of Morris \& Brady (1998), we note that having particles of a different density breaks the symmetry around the centre of the channel, such that the maximum in the particle volume fraction and in the velocity tends to occur above the centreline. Most importantly, the rapid shearing motion close to the bottom wall generates a large pressure (via the suspension temperature and $(2.4 c)$ for the particle-phase pressure) so that the region of low density near the wall can support the weight of the heavier, slower shearing region in the middle, and the system has an adverse density profile of heavier material over light, the driving force for instability. 

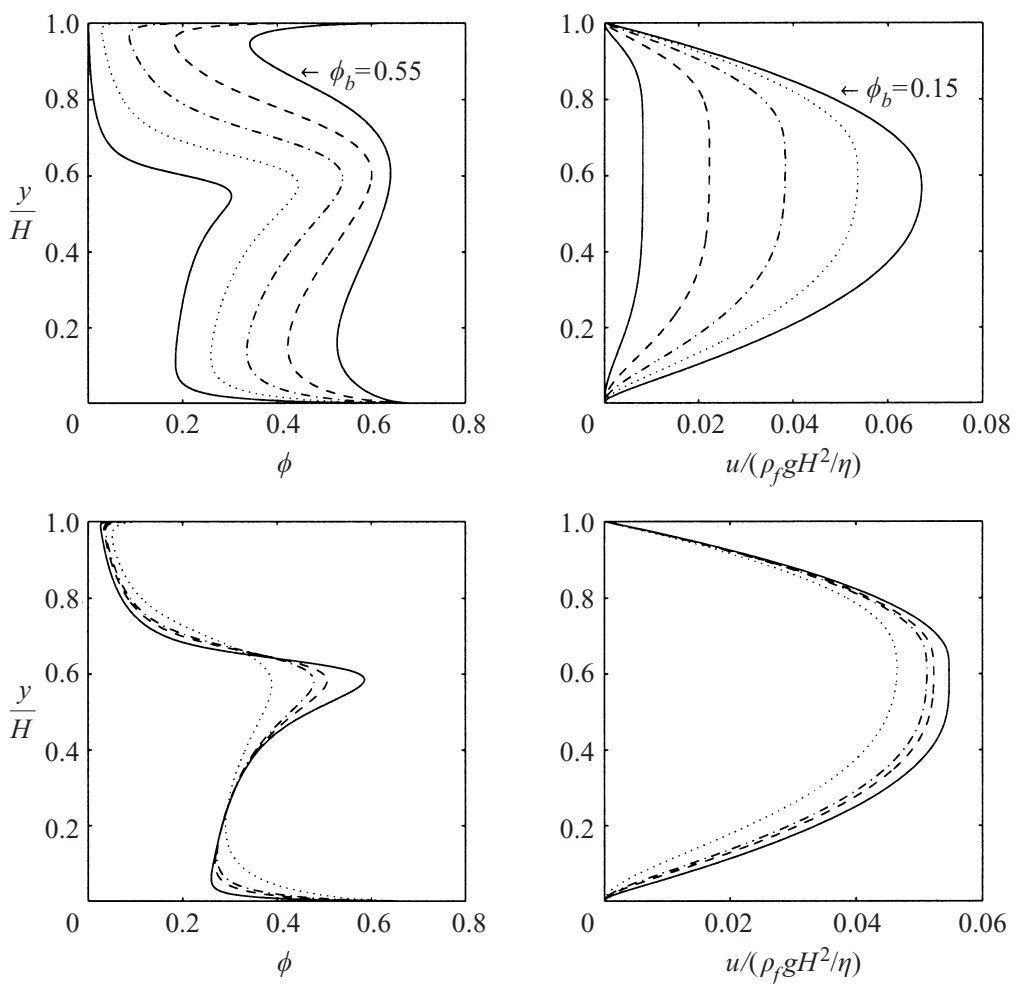

FIGURE 4. Base-state volume fraction and velocity profiles for $B=1$ and $\theta=45^{\circ}$. Top: $H / a=18.32$, with $\left.\phi_{b}=\{0.15(-), 0.25(\cdots), 0.35(-\cdot-\cdot-), 0.45(--)), 0.55(-)\right\}$. Bottom: $\phi_{b}=0.27$, with $H / a=\{10(\cdots), 20(-\cdot-\cdot-), 25(---), 50(-)\}$. The velocity increases with decreasing $\phi_{b}$ and increasing $H / a$. The density (volume fraction) profile decreases with decreasing $\phi_{b}$ and its maximum in the centre of the channel becomes more pronounced with increasing $H / a$.
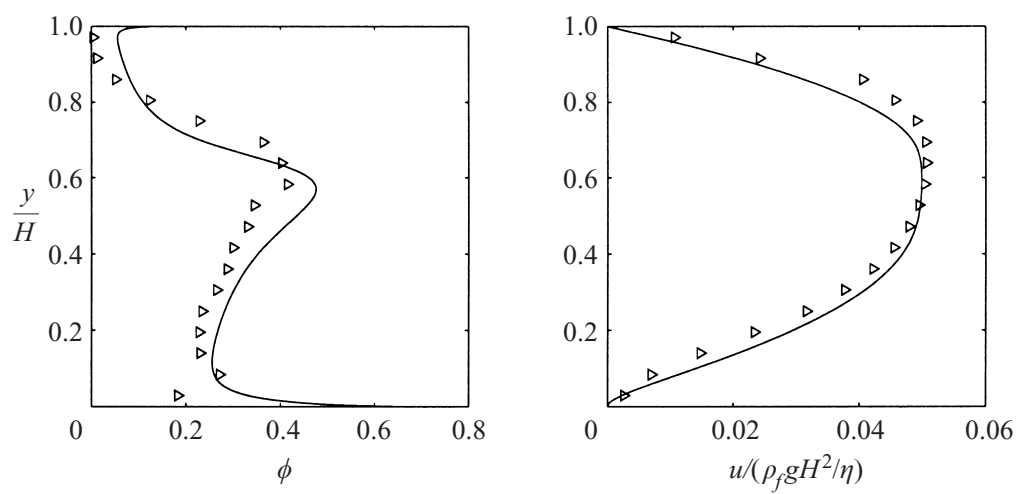

FIGURE 5. Base-state particle volume fraction and velocity profiles for $\phi_{b}=0.27, H / a=18.32$, $B=0.8$ and $\theta=45^{\circ}$. Comparison of model results (line) with Stokesian Dynamics simulations (triangles).

The results of the model compare favourably to Stokesian Dynamics simulations, as can be seen in figure 5 . These base state profiles may depend slightly upon the choice of model - for example, one could use the diffusive-flux model of Zhang \& Acrivos (1994). However, as shown in Fang et al. (2002), these differences are minor, and the inverted concentration profile, the important result, is maintained regardless 
of the model chosen. The mechanism of instability and all of the conclusions drawn here are therefore independent of the model.

\section{Linear stability analysis}

We perform a standard linear stability analysis of equations (2.1)-(2.3) to perturbations in the spanwise direction $z$ of the form $f^{\prime}(y)=\delta f(y) \mathrm{e}^{\mathrm{i} k z+s t}$, with $k$ the wavenumber and $s$ the growth rate. Here $\delta$ is the small amplitude of the perturbation. (Note that $f(y)$ denotes a general function, not to be confused with the hindered settling function $f(\phi)$.) All variables are non-dimensionalized as before. In solving the perturbed problem, all terms of $O\left(\epsilon^{2}, \delta^{2}\right)$ or smaller are neglected, and the linearized perturbation problem becomes

$$
\begin{gathered}
\mathrm{D}\left(\eta_{s}^{\circ} \mathrm{D} u\right)-\tilde{k}^{2} \eta_{s}^{\circ} u=-B \psi \sin \theta-\mathrm{D}\left(\eta_{s}^{\prime} \psi \mathrm{D} u^{\circ}\right), \\
\left(\mathrm{D}^{2}+\tilde{k}^{2}\right) \eta_{s}^{\circ}\left(\mathrm{D}^{2}+\tilde{k}^{2}\right) v-4 \tilde{k}^{2} \mathrm{D}\left(\eta_{s}^{\circ} \mathrm{D} v\right)=-B \psi \tilde{k}^{2} \cos \theta, \\
\tilde{s} \psi=-v \mathrm{D} \phi^{\circ}, \\
-\alpha^{\circ} T=\alpha^{\prime} T^{\circ} \psi-2 \eta_{p}^{\circ} \mathrm{D} u^{\circ} \mathrm{D} u-\eta_{p}^{\prime}\left(\mathrm{D} u^{\circ}\right) \psi,
\end{gathered}
$$

subject to the conditions

$$
\begin{gathered}
u=v=\mathrm{D} v=0 \quad \text { at } y=0 \text { and } y=1, \\
T=\frac{\eta_{p}^{\prime}}{\alpha} \psi\left(\mathrm{D} u^{\circ}\right)^{2}+2 \frac{\eta_{p}^{\circ}}{\alpha^{\circ}} \mathrm{D} u^{\circ} \mathrm{D} u \quad \text { at } y=0 \text { and } y=1, \\
\int_{0}^{1} \int_{-W / H}^{W / H} \psi \mathrm{d} y \mathrm{~d} z=0,
\end{gathered}
$$

where $\psi$ is the perturbation in volume fraction, $\mathrm{D}=\mathrm{d} / \mathrm{d} y,{ }^{\circ}$ denotes a base-state quantity and the' denotes differentiation with respect to the volume fraction. The dimensionless wavenumber and growth rate are $\tilde{k}=k H, \tilde{s}=s H / U_{c}=s /\left(\rho_{f} g H / \eta\right)$, respectively, and $W$ is the period of the perturbation in the $z$-direction.

In order to determine the growth rate $\tilde{s}(\tilde{k})$, we need only solve the eigenvalue problem obtained by combining $(4.1 b)$ and $(4.1 c)$ :

$$
\left(\mathrm{D}^{2}+\tilde{k}^{2}\right) \eta_{s}^{\circ}\left(\mathrm{D}^{2}+\tilde{k}^{2}\right) v-4 \tilde{k}^{2} \mathrm{D}\left(\eta_{s}^{\circ} \mathrm{D} v\right)=-\frac{B \tilde{k}^{2} \cos \theta \mathrm{D} \phi^{\circ}}{\tilde{s}} v
$$

with the boundary conditions on $v$ given by (4.2a). Equation (4.3) shows clearly that the driving force for the instability is the base-state density gradient $\mathrm{D} \phi^{\circ}=\mathrm{d} \phi^{\circ} / \mathrm{d} y$. When $\mathrm{d} \phi^{\circ} / \mathrm{d} y>0$ the system has an adverse density stratification and is unstable. Equation (4.3) is a fourth-order ordinary differential equation with variable coefficients and was solved numerically. Matlab proved quite helpful in this regard and the results corresponding to the base-state profiles given in the previous section are shown in figures 6 and 7.

In the sedimenting limit, $\theta \rightarrow 0$ or $B \rightarrow \infty$, the density gradient $\mathrm{D} \phi^{\circ}$ is negative throughout the channel, and the system is stable - light material over heavy. However, for intermediate values of $\theta\left(0^{\circ}<\theta<90^{\circ}\right)$ and $B$ there is always some heavier material above light, a system which is inherently unstable. In these regimes, the temporal growth rate of the instability is positive and any small perturbation would grow. The system is unstable for all wavenumbers, with a maximum growth rate at 

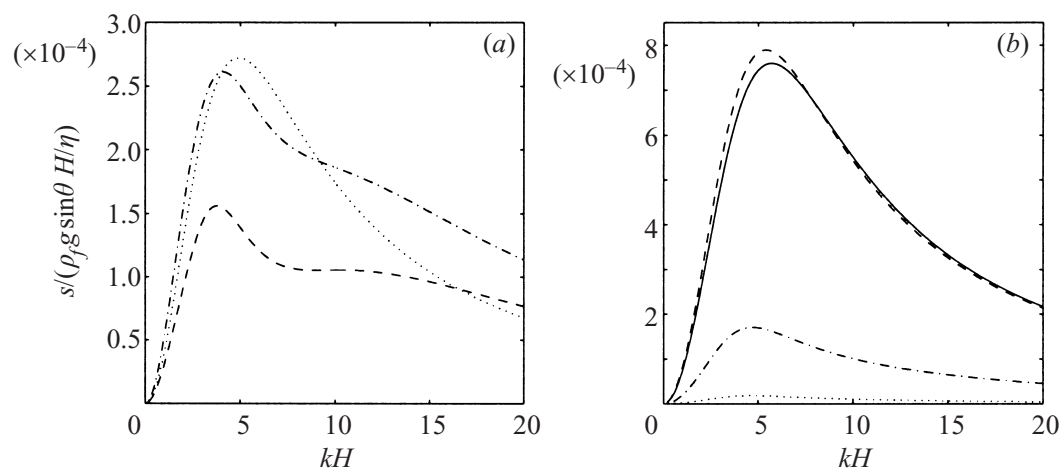

FIGURE 6. (a) Dimensionless temporal growth rate of the instability, $s /\left(\rho_{f} g \sin \theta H / \eta\right)$, as a function of dimensionless wavenumber $k H$ for $\phi_{b}=0.40, H / a=30.54$ and $B=1$ : $\theta=\left\{30^{\circ}(\cdots), 50^{\circ}(-\cdots-\cdot-), 70^{\circ}(--)\right\}$. Because here $s$ has been scaled with $\sin \theta$, the growth rate increases with decreasing $\theta$. If this scaling were removed, the growth rate would achieve a maximum at an intermediate value of $\theta$. (b) $\phi_{b}=0.27, H / a=18.32$ and $\theta=45^{\circ}$ : $B=\{0.01(\cdots), 0.1(-\cdot-\cdot-), 1(---), 1.4(-)\}$.
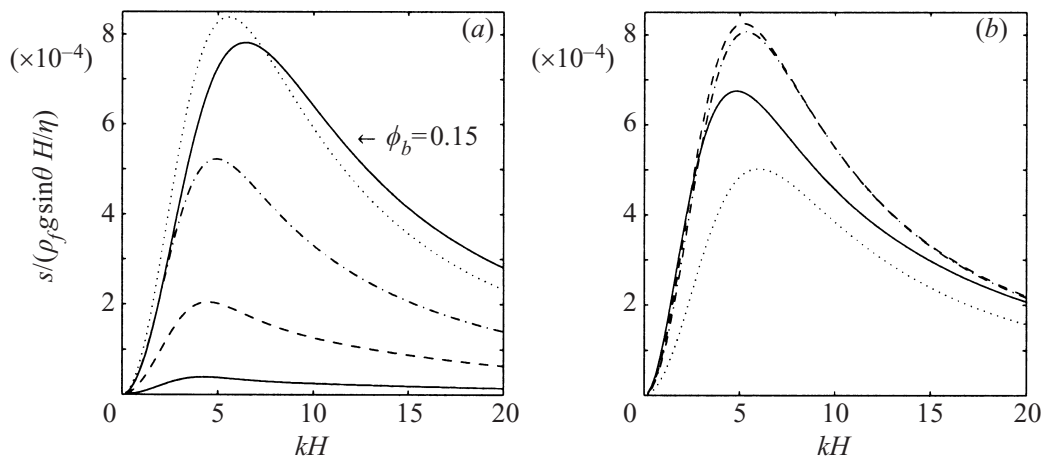

FiguRE 7. (a) Dimensionless temporal growth rate of the instability, $s /\left(\rho_{f} g \sin \theta H / \eta\right)$, as a function of dimensionless wavenumber $k H$ for $B=1, \theta=45^{\circ}, H / a=18.32$ and $\phi_{b}=\{0.15(-), 0.25(\cdots), 0.35(-\cdot-\cdot-), 0.45(---), 0.55(-)\}$. (b) $B=1, \theta=45^{\circ}$, $\phi_{b}=0.27$ and $H / a=\{10(\cdots), 20(-\cdot-\cdot-), 25(---), 50(-)\}$.

a wavelength of $O(H)$, the height of the layer. (From (4.3) one can see that in the low-wavenumber limit $(\tilde{k} \rightarrow 0) \tilde{s}$ grows as $\tilde{k}^{2}$, while in the high-wavenumber limit $(\tilde{k} \rightarrow \infty) \tilde{s}$ decays as $1 / \tilde{k}^{2}$ due to viscous damping.) Thus, one would expect patterns of this wavelength to form initially, which might then lead to the kinds of patterns seen in suspension and granular flows. (Note that, although the system is unstable as $\tilde{k} \rightarrow \infty$, when the wavelength is of order the particle size, $k a \sim O(1)$, the continuum model may no longer be appropriate and we could expect, therefore, a high- $\tilde{k}$ cutoff.)

The results shown in figure 6 illustrate the effect of varying $B$ and $\theta$ upon the growth rate of the instability. Increasing $B$ - increasing the relative density difference between the particles and fluid - causes the system to be more unstable, at least in the intermediate $B$ range. The instability of the system is also dependent upon $\theta$. Basically, the two extremes of inclination - horizontal and vertical - give two stability limits. In the limit $\theta \rightarrow 0^{\circ}$, the system moves towards sedimentation. In the limit $\theta \rightarrow 90^{\circ}$, there is no longer a component of gravity perpendicular to the walls, and, even though the density varies in the 'right' way for instability, the system is 
stable due to the lack of a driving force. As a result, the system is most unstable for angles that lie between these two extremes. Figure 7(a) illustrates the effect of varying the bulk volume fraction $\phi_{b}$. Increasing $\phi_{b}$ is similar to increasing $B-$ the system becomes slightly more stable for the higher bulk volume fractions. Figure 7(b) shows the effect of varying $H / a$, and it is seen that provided $H / a \gtrsim 10$ there is little effect of the particle size on the instability. (Note that $H / a$ must be large in order to treat the suspension as a continuum.) Indeed, to a first approximation the growth rate and most rapidly growing wavelength are roughly independent of particle size and the slight decrease in the growth rate for large $H / a$ is due to the high volume fraction near the centreline (cf. figure 4) which increases the local viscosity. It is important to note that the system is most unstable at intermediate values of the parameters - that is, when the system is at its most physically reasonable - not $\theta \rightarrow 0^{\circ}$ nor $\theta \rightarrow 90^{\circ}$, not $B \rightarrow 0$ nor $B \rightarrow \infty$, not $H / a \rightarrow 1$ nor $H / a \rightarrow \infty$ and not $\phi_{b} \rightarrow 0$ nor $\phi_{b} \rightarrow \phi_{m}$, but somewhere in-between.

\section{Conclusions}

For the problem of gravity-driven flow of non-neutrally buoyant suspensions, we report the existence of a maximum growth rate for spanwise instabilities on the order of the height of the channel, $H$. The instability is present whenever the flow-generated density gradient is aligned antiparallel to gravity. Although this unstable density gradient is generated only by flow, it shares some characteristics with the classical Rayleigh-Taylor instability - basically, having heavier material over light. The system is unstable to small perturbations over the range of the most physically reasonable particle-fluid density ratios and channel inclination angles, with the growth rate of the instability depending upon these parameters as well as on the bulk particle volume fraction and to a much lesser extent on the ratio of particle size to channel height. As seen in figures 6 and 7 the growth rate is small and therefore one would need a long channel $(L / H \sim O(1 / \widetilde{s}))$ in order to see the instability, suggesting that rotating drum experiments might be more desirable in practice. Also note that although the precise details of the growth rate, etc. depend on the constitutive relations used in the suspension-balance model, the basic instability mechanism does not.

Although we are not aware of any heavy viscous suspension experiments with which to compare, the experiments of Forterre \& Pouliquen (2001) for the stability of granular flow down an inclined plane are qualitatively similar to the system examined in this work. Rapid granular flows display the same flow-induced adverse density profile and therefore should be subject to the same instability. Indeed, the experiments of Forterre \& Pouliquen (2001) showed the formation of spanwise patterns whose wavelength was on the order of $3 h$, where $h$ was the height of their granular layer. It is interesting to note that for the current work, the most unstable wavelength tends to be approximately $1.2 H$ for most parameter sets (corresponding to $\tilde{k} \sim 5$ ), and since the channel height is approximately twice that of the suspension layer, $H \approx 2 h$, the most unstable wavelength is approximately $2.4 h$, quite close to the observed granular flow instability wavelength.

In addition to the extra inertial effects associated with granular flows (note, however, that in the steady unidirectional base state, inertia is not important), the stability of suspensions and granular flows may also be affected by the presence of normal stress differences (Brady \& Carpen 2002), which were not included in the present analysis. Normal stresses can be expected to enhance the intriguing behaviour of these systems and might prove interesting to investigate. 
Finally, the gravity-driven flow investigated here may also be unstable to longitudinal perturbations - in the flow direction, for example as seen in the case of stratified flow with a free surface (Plaschko \& Schaflinger 1998) - perturbations whose form will now be complex: a travelling wave in addition to an exponentially growing part.

The authors would like to thank Dr Z. Fang for his help with the numerical solution of the base-state problem as well as for providing the Stokesian Dynamics simulation results.

\section{REFERENCES}

BRAdy, J. F. \& CARPEN, I. C. 2002 Second normal stress jump instability in non-Newtonian fluids. J. Non-Newtonian Fluid Mech. 102, 219-232.

Brady, J. F. \& Morris, J. F. 1997 Microstructure of strongly sheared suspensions and its impact on rheology and diffusion. J. Fluid Mech. 348, 103-139.

Fang, Z., Mammoli, A. A., Brady, J. F., Ingber, M. S., Mondy, L. A. \& Graham, A. L. 2002 Flow-aligned tensor models for suspension flows. Intl J. Multiphase Flow 28, 137-166.

Forterre, Y. \& Pouliquen, O. 2001 Longitudinal vortices in granular flows. Phys. Rev. Lett. 86, $5886-5889$.

Fried, E., Shen, A. Q. \& Thoroddsen, S. T. 1998 Wave patterns in a thin layer of sand within a rotating horizontal cylinder. Phys. Fluids 10, 10-12.

Hill, K. M. \& KaKalios, J. 1995 Reversible axial segregation of rotating granular media. Phys. Rev. E 52, 4393-4400.

Jaeger, H. M., Nagel, S. R. \& Behringer, R. P. $1996 a$ The physics of granular materials. Phys. Today 49, 32-38.

Jaeger, H. M., NAgel, S. R. \& Behringer, R. P. $1996 b$ Granular solids, liquids, and gases. Rev. Mod. Phys. 68, 1259-1273.

Lange, A., Schröter, M., Scherer, M. A., Engel, A. \& Rehberg, I. 1998 Fingering instability in a water-sand mixture. Eur. Phys. J. B 4, 475-484.

Morris, J. F. \& Brady, J. F. 1998 Pressure-driven flow of a suspension: buoyancy effects. Intl J. Multiphase Flow 24, 105-130.

NotT, P. R. \& Brady, J. F. 1994 Pressure-driven flow of suspensions: simulation and theory. J. Fluid Mech. 275, 157-199.

Plaschko, P. \& Schaflinger, U. 1998 Absolute instabilities in two-layer gravity driven film flow with a free surface. Z. Angew. Math. Phys. 49, 194-204.

Pouliquen, O., Delour, J. \& Savage, S. B. 1997 Fingering in granular flows. Nature 386, 816-817.

Savage, S. B. 1984 The mechanics of rapid granular flows. Adv. Appl. Mech. 24, 289-366.

Thomas, P. J., Riddell, G. D., Kooner, S. \& King, G. P. 2001 Fine structure of granular banding in two-phase rimming flow. Phys. Fluids 13, 2720-2723.

Tirumkudulu, M., Mileo, A. \& ACrivos, A. 2000 Particle segregation in monodisperse sheared suspensions in a partially filled rotating horizontal cylinder. Phys. Fluids 12, 1615-1618.

Umbanhowar, P. B., Melo, F. \& Swinney, H. L. 1996 Localized excitations in a vertically vibrated granular layer. Nature 382, 793-796.

Völtz, C., Pesch, W. \& Rehberg, I. 2001 Rayleigh-Taylor instability in a sedimenting suspension. Phys. Rev. E 65, 011404.

ZHANG, K. \& ACRIVOS, A. 1994 Viscous resuspension in fully developed laminar pipe flows. Intl J. Multiphase Flow 20, 579-591. 